\title{
Elevated lipoprotein(a) levels predict cardiovascular disease in type 2 diabetes mellitus: a 10-year prospective cohort study
}

Tae-Seok Lim ${ }^{1}$, Jae-Seung Yun ${ }^{1}$, Seon-Ah Cha ${ }^{1}$, Ki-Ho Song ${ }^{1}, \mathrm{Ki}-$ Dong Yoo $^{2}$, Yu-Bae Ahn ${ }^{1}$, Yong-Moon Park ${ }^{3}$, and Seung-Hyun $\mathrm{Ko}^{1}$

Divisions of ${ }^{1}$ Endocrinology and Metabolism and ${ }^{2}$ Cardiology, Department of Internal Medicine, College of Medicine, The Catholic University of Korea, Seoul, Korea; ${ }^{3}$ Department of Epidemiology and Biostatistics, Arnold School of Public Health, University of South Carolina, Columbia, SC, USA

\author{
Received: January 29, 2016 \\ Revised : April 4, 2016 \\ Accepted: May 5, 2016

\section{Correspondence to} \\ Seung-Hyun Ko, M.D. \\ Division of Endocrinology and \\ Metabolism, Department of In- \\ ternal Medicine, College of Med- \\ icine, St. Vincent's Hospital, The \\ Catholic University of Korea, 93 \\ Jungbu-daero, Paldal-gu, Suwon \\ 16247, Korea \\ Tel: $+82-31-249-8174$ \\ Fax: $+82-31-253-8898$ \\ E-mail: kosh@catholic.ac.kr
}

Background/Aims: Elevated lipoprotein(a) (Lp[a]) level is known to be a risk factor for cardiovascular disease (CVD). However, the data that has been reported on the association between the Lp(a) level and CVD in type 2 diabetes has been limited and incoherent. The aim of this study was to investigate the relationship between the $\mathrm{Lp}(\mathrm{a})$ concentration and new onset CVD in type 2 diabetes.

Methods: From March 2003 to December 2004, patients with type 2 diabetes without a prior history of CVD were consecutively enrolled. CVD was defined as the occurrence of coronary artery disease or ischemic stroke. Cox proportional hazards models were used to identify the associations between the Lp(a) and CVD after adjusting for confounding variables.

Results: Of the 1,183 patients who were enrolled, 833 participants were evaluated with a median follow-up time of 11.1 years. A total of 202 participants were diagnosed with CVD (24.2\%). The median Lp(a) level for 1st and 4 th quartile group was 5.4 (3.5 to 7.1 ) and $55.7 \mathrm{mg} / \mathrm{dL}$ (43.1 to 75.3). Compared with patients without CVD, those with CVD were older, had a longer duration of diabetes and hypertension, and used more insulin and angiotensin converting enzyme inhibitors/angiotensin receptor blockers at baseline. A Cox hazard regression analysis revealed that the development of CVD was significantly associated with serum Lp(a) level (hazard ratio, 1.92; 95\% confidence interval [CI], 1.26 to $2.92 ; p<0.001$, comparing the 4th vs. 1st quartile of Lp[a]).

Conclusions: Elevated Lp(a) level was an independent predictable risk factor for CVD in type 2 diabetes. Other cardiovascular risk factors should be treated more intensively in type 2 diabetic patients with high Lp(a) levels.

Keywords: Lipoprotein(a); Cardiovascular diseases; Diabetes mellitus, type 2

\section{INTRODUCTION}

Cardiovascular disease (CVD) is a major cause of death worldwide and is the second leading cause of death in Korea. In type 2 diabetes, the prevalence of CVD is 2- to 4-fold higher than in the general population [1-3]. Be- cause of the clinical importance of CVD and the related morbidity and mortality in type 2 diabetes, predictable risk engines based on large epidemiologic studies have been suggested to identify the risk factors $[4,5]$. Nevertheless, most of the previous studies were performed using only conventional risk factors (hypertension, lipid 
abnormalities, smoking, and obesity) for explaining the higher incidence of CVD in patients with type 2 diabetes $[1-3]$. Therefore, beyond the known modifiable risk factors, identification of another biomarker might be helpful for early detection and prediction of the future development of CVD in patients with type 2 diabetes. Moreover, a new biomarker may help to attenuate the health burden related to morbidity and mortality of CVD in patients with type 2 diabetes.

Lipoprotein(a) (Lp[a]) is a low density lipoprotein (LDL)-like substance composed of a cholesterol-laden LDL particle covalently bound to a plasminogen-like glycoprotein known as apolipoprotein(a) (apo[a]) [6]. Because apo(a) shows a strong sequence homology with plasminogen, $\mathrm{Lp}(\mathrm{a})$ has been considered a link between atherosclerosis and thrombosis [7]. Although its physiologic function remains elusive, recent large prospective studies and meta-analyses have documented the association of Lp(a) with an increased risk of CVD in the general population [8-10]. In the prospective meta-analysis studies of Emerging Risk Factors Collaboration et al. [8], the risk ratio for CVD per 3.5-fold higher Lp(a) levels was 1.16 (95\% confidence interval [CI], 1.11 to 1.22). Recently, Kamstrup and Nordestgaard [11] revealed that Lp(a) was inversely associated with the new onset of type 2 diabetes. However, several studies reviewing the association between Lp(a) concentration and CVD in type 2 diabetes reported inconsistent findings [12-17]. Moreover, the clinical implication of Lp(a) in CVD and the relationship between Lp(a) level and future development of CVD is unknown in patients with type 2 diabetes.

Therefore, the purpose of this long-term prospective observational cohort study was to investigate the association between $\mathrm{Lp}(\mathrm{a})$ level and CVD in type 2 diabetes.

\section{METHODS}

\section{Study population}

From January 2003 to December 2004, 1,183 patients with type 2 diabetes aged 25 to 75 years were consecutively enrolled and received follow-up care from January 2013 to December 2014 at the University-Affiliated Diabetes Center of St. Vincent's Hospital in South Korea (Fig. 1). Eligible participants were free from a history of CVD, type 1 diabetes, gestational diabetes, or hormone replacement therapy, such as with estrogens or progesterone; drugs interfering with $\mathrm{Lp}(\mathrm{a})$ metabolism such as niacin and chronic use of steroid; or any severe illness, such as heart failure, liver cirrhosis, alcoholism, thyroid disease, severe infection, acute illness, or malignancy. The study was approved by the Catholic Medical Center Ethics Committee and conducted in accordance with the Declaration of Helsinki. Written informed consents were obtained from all participants.

\section{Study method}

A detailed questionnaire was obtained for participant information, including the medical history, current cigarette smoking status, diabetes duration [18] and use of medications. Hypertension was defined as a systolic blood pressure $\geq 140 \mathrm{mmHg}$ or diastolic blood pressure $\geq 90 \mathrm{mmHg}$ or use of antihypertensive medications [19]. Blood samples were collected after fasting for 8 to 12 hours. The glycated hemoglobin ( $\mathrm{HbAlc})$ levels were measured by high-performance liquid chromatography every 6 months. The estimated glomerular filtration rate (eGFR) was used to determine the chronic kidney disease classification using the 4-component Modification of Diet in Renal Disease equation [20]. Diabetic nephropathy was diagnosed if a patient showed microalbuminuria (30 to $300 \mathrm{mg} /$ day) or macroalbuminuria ( $\geq$ $300 \mathrm{mg} /$ day). The urinary albumin excretion (UAE) rate was measured from a 24-hour urine collection using immunoturbidimetry (Eiken, Tokyo, Japan). The serum $\mathrm{Lp}$ (a) concentration was identified by a one-step sandwich enzyme-linked immunoassay (TintElize Lp[a] kit, Biopool AB, Umea, Sweden). The intra- and interassay

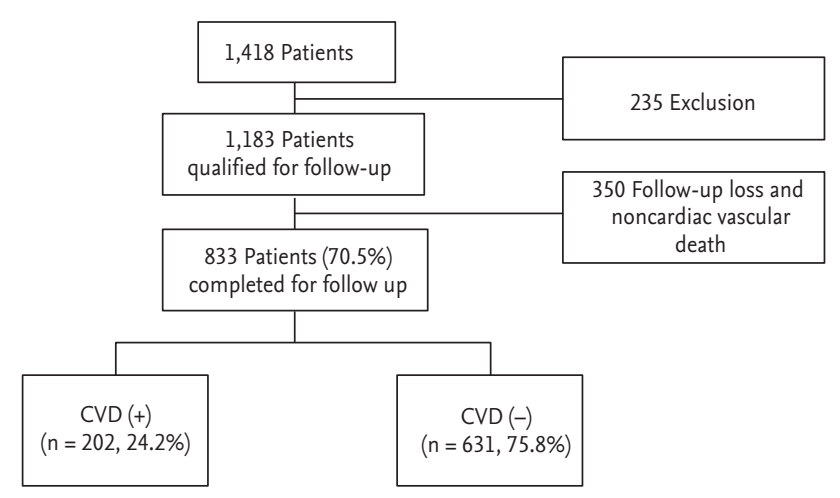

Figure 1. Study design summarizing and follow-up. CVD, cardiovascular disease. 
coefficients of variation were $4.5 \%$ and $6.7 \%$, respectively. Total cholesterol and LDL-cholesterol (LDL-C) levels were adjusted for the $\mathrm{Lp}(\mathrm{a})$ concentration according to the modified version of the formula which was published previously [21]. The modified formula is as follows: Lp(a)-corrected LDL-C = total cholesterol - high density lipoprotein cholesterol (HDL-C) - (triglycerides / 5) $-[\operatorname{Lp}(\mathrm{a}) \times 0.45]$.

\section{Definition of CVD}

CVD was defined as a diagnosis of coronary artery disease $(\mathrm{CAD})$ or stroke $[2,8]$. CAD was defined as either a diagnosis of angina pectoris, myocardial infarction, or coronary revascularization (coronary bypass surgery or coronary angioplasty). Stroke manifestation included transient ischemic attack (TIA) or cerebral infarction. Follow-up visits were performed every 3 to 4 months, and we verified the onset of CVD by hospital records. Diagnosis of CVD was confirmed by specialists including a cardiologist, a neurologist, and a neurosurgeon. Cause and time of death were obtained from the hospital record or by telephone call when they did not appear for follow-up.

\section{Statistical analysis}

All data are reported as the mean $\pm \mathrm{SD}$, median (interquartile range [IQR]) or number (percentage). The categorical variables were tested using chi-square tests, and the continuous variables were tested using the independent Student $t$ tests. As the Lp(a) concentration showed a highly skewed distribution, the Wilcoxon rank sum test was performed. Univariable and multivariable Cox proportional hazards models were used to identify the associations between the Lp(a) and CVD after adjusting for clinical characteristics (gender, age, duration of diabetes, hypertension, smoking, body mass index [BMI], diabetic nephropathy, mean $\mathrm{HbAlc}$ level, fasting plasma glucose [FPG], eGFR, Lp[a] corrected LDL-C, insulin treatment, the use of angiotensin converting enzyme inhibitor/angiotensin receptor blockers [ACEi/ARBs], acetylsalicylic acid, or statins). The proportional hazards assumption was confirmed using log-minus log-survival plots. Hazard ratios (HRs) were calculated and the results were reported as HRs and 95\% CI. SAS version 9.3 (SAS Institute Inc., Cary, NC, USA) was used for statistical analyses. A p $<0.05$ was considered significant.

\section{RESULTS}

During 11.1 years of follow-up, 833 participants (70.5\%) were evaluated (Fig. 1) and 79 participants (9.5\%) died (12 patients died due to CVD event) in 1,183 participants. A total of 503 participants (60.4\%) were women, and the mean age and diabetes duration was $55.2 \pm 9.9$ and $7.8 \pm$ 6.3 years, respectively. During the follow-up period, 202 participants $(24.2 \%)$ were newly diagnosed with CVD; 114 cases (13.7\%) were CAD event, 97 cases (11.6\%) were stroke (including TIA), and nine participants ( $1 \%$ ) were diagnosed with both CAD and stroke.

Compared with patients without CVD, those with CVD were older, more likely to be female, had a longer duration of diabetes, had a higher frequency of hypertension and diabetic nephropathy, and used more insulin and ACEi/ARBs at baseline (Table 1). The group with CVD had a higher mean HbAic during the follow-up than those without CVD $(8.18 \% \pm 1.21 \%$ vs. $8.60 \% \pm 1.38 \%, p<$ $0.001)$. Of the 115 patients who had a mean $\mathrm{HbA1c}<7.0 \%$ during the observation period, 17 patients (14.8\%) developed CVD at the end of the study. However, of the 717 patients who had mean HbAıc $>9.0 \%$ during the study period, 184 patients $(25.7 \%)$ developed CVD $(p=0.011)$.

In laboratory findings at baseline, HbArc level, total cholesterol, and LDL-C levels were significantly higher in the CVD group. In particular, Lp(a) levels were different between subjects with CVD and without CVD (25.6 $\mathrm{mg} / \mathrm{dL}$ vs. $15.2 \mathrm{mg} / \mathrm{dL}, p<0.001$ ) (Table 1). The median baseline $\mathrm{Lp}(\mathrm{a})$ level of this cohort was $16.7 \mathrm{mg} / \mathrm{dL}$, and the distribution of $\mathrm{Lp}(\mathrm{a})$ levels was skewed, ranging from 0.35 to $261.0 \mathrm{mg} / \mathrm{dL}$. The median Lp(a) level in females was higher than that in males $(20.3 \mathrm{mg} / \mathrm{dL}$ [IQR, 10.5 to 37.6 ] vs. $13.1 \mathrm{mg} / \mathrm{dL}$ [IQR, 7.2 to 29.5$], p<0.001$ ).

The median $\mathrm{Lp}(\mathrm{a})$ level for each quartile group was 5.4 $\mathrm{mg} / \mathrm{dL}$ (IQR, 3.5 to 7.1), $12.1 \mathrm{mg} / \mathrm{dL}$ (IQR, 10.3 to 14.7), 24.0 $\mathrm{mg} / \mathrm{dL}$ (IQR, 20.5 to 29.3), and $55.7 \mathrm{mg} / \mathrm{dL}$ (IQR, 43.1 to 75.3 ) (Table 2). The patients with elevated Lp(a) levels tended to be older and have a longer diabetes duration. In addition, FPG, HbAıc, total cholesterol, LDL-C, and UAE levels were higher in the patients with elevated $L p(a)$ concentrations (Table 2). Triglycerides, Lp(a) corrected total cholesterol, Lp(a) corrected-LDL-C, and eGFR were inversely associated with $\mathrm{Lp}(\mathrm{a})$ concentrations. Other baseline characteristics such as hypertension, diabetic nephropathy, BMI, smoking, HDL-C, and use of ACEi/ 
Table 1. Baseline characteristics in type 2 diabetes patients who developed CVD events compared with those who did not

\begin{tabular}{|c|c|c|c|c|}
\hline Characteristic & Total & $\operatorname{CVD}(-)$ & $\operatorname{CVD}(+)$ & $p$ value \\
\hline Number & 833 & 631 & 202 & \\
\hline $\mathrm{Lp}(\mathrm{a}), \mathrm{mg} / \mathrm{dL}$ & $16.7(8.3-35.1)$ & $15.2(7.8-31.6)$ & $25 \cdot 6(5 \cdot 9-35 \cdot 3)$ & $<0.001$ \\
\hline Female sex & $503(60.4)$ & $365(57.8)$ & $138(68.3)$ & 0.008 \\
\hline Age, yr & $55.2 \pm 9.9$ & $53.8 \pm 9.8$ & $59.7 \pm 8.8$ & $<0.001$ \\
\hline Diabetes duration, yr & $7.8 \pm 6.3$ & $7.1 \pm 6.0$ & $10.3 \pm 6.9$ & $<0.001$ \\
\hline Hypertension & $384(46.1)$ & $264(41.8)$ & $120(59.4)$ & $<0.001$ \\
\hline Body mass index, $\mathrm{kg} / \mathrm{m}^{2}$ & $24 \cdot 7 \pm 3 \cdot 4$ & $24 \cdot 6 \pm 3 \cdot 3$ & $25.1 \pm 3.5$ & 0.064 \\
\hline Smoking & $191(22.9)$ & $144(22.8)$ & $47(23 \cdot 3)$ & 0.895 \\
\hline Insulin & $211(25 \cdot 3)$ & $129(20.4)$ & $82(40.6)$ & $<0.001$ \\
\hline ACEi/ARBs & $248(29.8)$ & $174(27 \cdot 6)$ & $74(36.6)$ & 0.014 \\
\hline Statin & $97(11.6)$ & $71(11.3)$ & $26(12.9)$ & 0.532 \\
\hline Acetylsalicylic acid & $58(7.0)$ & $32(5 \cdot 1)$ & $26(12.9)$ & $<0.001$ \\
\hline Diabetic nephropathy & $182(21.8)$ & $127(20.1)$ & $55(27.2)$ & 0.034 \\
\hline \multicolumn{5}{|c|}{ Laboratory finding at baseline } \\
\hline FPG, mg/dL & $176.3 \pm 64.9$ & $174 \cdot 3 \pm 63 \cdot 4$ & $182.7 \pm 69.2$ & 0.109 \\
\hline $\mathrm{eGFR}, \mathrm{mL} / \mathrm{min} / 1.73 \mathrm{~m}^{2}$ & $93.2 \pm 16.7$ & $94.8 \pm 16.1$ & $88.2 \pm 17.3$ & $<0.001$ \\
\hline Baseline HbAıc, \% & $8.9 \pm 2.1$ & $8.8 \pm 2.0$ & $9.2 \pm 2.1$ & 0.016 \\
\hline Total cholesterol, mg/dL & $183.6 \pm 36.7$ & $181.5 \pm 36.1$ & $189.8 \pm 38.0$ & 0.005 \\
\hline Triglyceride, mg/dL & $160.4 \pm 104.7$ & $159.2 \pm 106.0$ & $164 \cdot 3 \pm 100.8$ & 0.547 \\
\hline HDL-C, mg/dL & $43 \cdot 3 \pm 10.5$ & $43.3 \pm 10.1$ & $43.3 \pm 11.9$ & 0.989 \\
\hline LDL-C, mg/dL & $108.2 \pm 32.7$ & $106.6 \pm 32.3$ & $113.2 \pm 33.7$ & 0.013 \\
\hline Lp(a) corrected TC & $172.1 \pm 37.5$ & $170.9 \pm 37.1$ & $175.6 \pm 38.8$ & 0.128 \\
\hline Lp(a) corrected LDL-C & $96.7 \pm 32.6$ & $95.8 \pm 32.1$ & $99.4 \pm 34.1$ & 0.171 \\
\hline $\mathrm{UAE}, \mathrm{mg} /$ day & $10.0(5.6-28.0)$ & $10.0(5 \cdot 4-25.1)$ & $10.0(5 \cdot 9-35 \cdot 3)$ & 0.361 \\
\hline
\end{tabular}

Values are presented as median (interquartile range), number (\%), or mean \pm SD.

CVD, cardiovascular disease; Lp(a), lipoprotein(a); ACEi, angiotensin converting enzyme inhibitor; ARB, angiotensin receptor blocker; FPG, fasting plasma glucose; eGFR, estimated glomerular filtration rate; HbA1c, glycated hemoglobin; HDL-C, high density lipoprotein cholesterol; LDL-C, low density lipoprotein cholesterol; TC, total cholesterol; UAE, urinary albumin excretion.

ARBs, insulin, statins, and acetylsalicylic acid showed no significant differences among each tertile group (Table 2).

In univariable Cox regression analysis, female gender, old age, diabetes duration, hypertension, Lp(a) corrected LDL-C level, mean HbA1c, diabetic nephropathy, and use of insulin, ACEi/ARB, and acetylsalicylic acid were significant variables in patients with CVD. The HR of CVD development was 2.37 (95\% CI, 1.59 to $3.55 ; p<0.001$ ) for the top versus bottom quartile of Lp(a) (Table 3). In the Cox hazard logistic regression analysis, after adjusting for multiple variable factors (age, gender, diabetes duration, hypertension, smoking history, BMI, mean HbAlc, diabetic nephropathy, eGFR, Lp[a] corrected
LDL-C, and use of insulin, ACEi/ARBs, statins, and acetylsalicylic acid), the highest quartile of $\mathrm{Lp}(\mathrm{a})$ level tended to increase the risk of CVD (HR, 1.92; 95\% CI, 1.26 to 2.92; $p<0.001$ ) (Table 4). The result from an analysis of log-transformed Lp(a) level showed similar association between Lp(a) and CVD (HR, 2.10; 95\% CI, 1.42 to 3.11; p $<0.001$ per log increment in Lp[a] concentration). We showed multivariable Cox proportional hazards model for heart disease and stroke (Supplementary Table 1), and the figure of Kaplan-Meier curve of CVD events according to the quartiles of $\mathrm{LP}(\mathrm{a})$ (Supplementary Fig. 1) in supplementary information. 
Table 2. Descriptive characteristics by lipoprotein(a) level at baseline examination

\begin{tabular}{|c|c|c|c|c|c|}
\hline \multirow{2}{*}{ Characteristic } & \multicolumn{4}{|c|}{$\operatorname{Lp}(\mathrm{a})$} & \multirow{2}{*}{$p$ for trend } \\
\hline & 1st Quartile & 2nd Quartile & 3rd Quartile & 4th Quartile & \\
\hline Number & 207 & 209 & 209 & 208 & \\
\hline $\mathrm{Lp}(\mathrm{a}), \mathrm{mg} / \mathrm{dL}$ & $5 \cdot 4(3 \cdot 5-7 \cdot 1)$ & $12.1(10.3-14.7)$ & $24.0\left(20.5^{-29.3)}\right.$ & $55.7(43.1-75 \cdot 3)$ & $<0.001$ \\
\hline Female sex & $100(48.3)$ & $124(59 \cdot 3)$ & $139(66.5)$ & $140(67 \cdot 3)$ & $<0.001$ \\
\hline Age, yr & $53 \cdot 5 \pm 9.6$ & $56.0 \pm 9.8$ & $55.8 \pm 10.1$ & $55 \cdot 7 \pm 10.1$ & 0.038 \\
\hline Diabetes duration, yr & $6.5 \pm 5.2$ & $8.0 \pm 6.4$ & $8.4 \pm 6.8$ & $8.4 \pm 6.8$ & 0.002 \\
\hline Hypertension & $89(43.0)$ & $102(48.8)$ & $99(47.4)$ & $94(45 \cdot 2)$ & 0.741 \\
\hline Body mass index, $\mathrm{kg} / \mathrm{m}^{2}$ & $24 \cdot 7 \pm 3 \cdot 4$ & $24 \cdot 7 \pm 3 \cdot 3$ & $24 \cdot 7 \pm 3 \cdot 3$ & $24.8 \pm 3.4$ & 0.794 \\
\hline Smoking & $60(29.0)$ & $43(20.6)$ & $41(19.6)$ & $47(22.6)$ & 0.124 \\
\hline Insulin & $47(22.7)$ & $47(22.5)$ & $57(27 \cdot 3)$ & $60(28.8)$ & 0.085 \\
\hline ACEi/ARBs & $58(28.0)$ & $58(27.8)$ & $62(29.7)$ & $70(33.7)$ & 0.185 \\
\hline Statin & $20(9.7)$ & $27(12.9)$ & $16(7 \cdot 7)$ & $34(16.3)$ & 0.138 \\
\hline Acetylsalicylic acid & $14(6.8)$ & $15(7.2)$ & $12(5 \cdot 7)$ & $17(8.2)$ & 0.724 \\
\hline Diabetic nephropathy & $50(24.2)$ & $50(23.9)$ & $35(16.7)$ & $47(22.6)$ & 0.355 \\
\hline \multicolumn{6}{|c|}{ Laboratory finding at baseline } \\
\hline FPG, mg/dL & $174 \cdot 3 \pm 66.5$ & $167.6 \pm 59.0$ & $175 \cdot 7 \pm 61.4$ & $187.7 \pm 71.0$ & 0.016 \\
\hline $\mathrm{eGFR}, \mathrm{mL} / \mathrm{min} / 1.73 \mathrm{~m}^{2}$ & $96.1 \pm 15.7$ & $92.2 \pm 15.8$ & $92.4 \pm 17.0$ & $92.1 \pm 17.8$ & 0.020 \\
\hline Baseline HbAıc, \% & $8.5 \pm 1.9$ & $8.7 \pm 1.9$ & $8.8 \pm 2.0$ & $9.5 \pm 2.3$ & $<0.001$ \\
\hline Total cholesterol, mg/dL & $178.2 \pm 36.3$ & $185 \cdot 3 \pm 35 \cdot 8$ & $180.7 \pm 35.2$ & $190.0 \pm 38.6$ & 0.007 \\
\hline Triglyceride, mg/dL & $171.2 \pm 119.4$ & $162.4 \pm 101.8$ & $157.7 \pm 110.4$ & $157 \cdot 7 \pm 110.4$ & 0.039 \\
\hline HDL-C, mg/dL & $43.6 \pm 10.7$ & $43.2 \pm 9.7$ & $43.2 \pm 10.6$ & $43 \cdot 3 \pm 11.2$ & 0.720 \\
\hline LDL-C, mg/dL & $99.9 \pm 31.4$ & $109.6 \pm 31.3$ & $106.6 \pm 31.4$ & $116.7 \pm 34.9$ & $<0.001$ \\
\hline $\mathrm{Lp}(\mathrm{a})$ corrected TC & $175 \cdot 5 \pm 36.4$ & $179.8 \pm 35.8$ & $170.7 \pm 37.2$ & $162.3 \pm 38.7$ & $<0.001$ \\
\hline Lp(a) corrected LDL-C & $97.7 \pm 31.8$ & $104.1 \pm 31.3$ & $96.0 \pm 31.4$ & $89.0 \pm 34.2$ & 0.001 \\
\hline UAE, mg/day & $10.0(5.2-30.0)$ & $10.1(6.2-30.0)$ & $10.0(5.0-21.8)$ & $10.9(5.9-29.1)$ & 0.004 \\
\hline
\end{tabular}

Values are presented as median (interquartile range), number (\%), or mean \pm SD.

Lp(a), lipoprotein(a); ACEi, angiotensin converting enzyme inhibitor; ARB, angiotensin receptor blocker; FPG, fasting plasma glucose; eGFR, estimated glomerular filtration rate; HbAıc, glycated hemoglobin; HDL-C, high density lipoprotein cholesterol; LDL-C, low density lipoprotein cholesterol; TC, total cholesterol; UAE, urinary albumin excretion.

\section{DISCUSSION}

In this long-term prospective cohort study in type 2 diabetes patients without a prior CVD history, we found a significant association between Lp(a) level and development of CVD. Our findings suggested that the elevated Lp(a) concentration (highest quartile) was an independent risk factor for CVD in type 2 diabetes.

Many prospective studies and meta-analyses have indicated that $\mathrm{Lp}(\mathrm{a})$ is an independent predictor of CVD after adjustment for causal risk factors in the general population [8-10], as follows. A meta-analysis from 36 long-term prospective studies including 126,634 subjects found that there were continuous, independent, and modest associations between $\mathrm{Lp}(\mathrm{a})$ levels and risk of CAD and stroke (adjusted risk ratio for CAD was 1.16 [95\% CI, 1.11 to 1.22] per one SD increase) [8]. Kamstrup et al. [9] published data from the Copenhagen Heart Study comprising approximately 40,000 individuals in the general population. They showed consistent findings with a causal association between elevated Lp(a) levels, KIV-2 genotype, and increased risk of myocardial infarction. In patients with a previous CAD history, elevated Lp(a) was associated with worse outcomes (both CAD 
Table 3. Univariable Cox proportional hazards model for cardiovascular disease

\begin{tabular}{|c|c|c|}
\hline Variable & Hazard ratio (95\% CI) & $p$ value \\
\hline Female sex & $1.46(1.08-1.96)$ & 0.013 \\
\hline Age (per 10 years) & $1.80\left(1.55^{-2.10}\right)$ & $<0.001$ \\
\hline Diabetes duration, yr & & $<0.001$ \\
\hline$<5$ & 1.00 & \\
\hline 5 to $<10$ & $1.25(0.83-1.88)$ & 0.280 \\
\hline$\geq 10$ & $2.65(1.91-3.68)$ & $<0.001$ \\
\hline Hypertension (yes vs. no) & $1.91(1.44-2.53)$ & $<0.001$ \\
\hline Smoking (yes vs. no) & $1.05(0.76-1.45)$ & 0.788 \\
\hline Insulin use (yes vs. no) & $2.23(1.68-2.95)$ & $<0.001$ \\
\hline ACEi/ARBs (yes vs. no) & $1.44(1.08-1.92)$ & 0.012 \\
\hline Statin (yes vs. no) & $1.17(0.77-1.76)$ & 0.464 \\
\hline Acetylsalicylic acid (yes vs. no) & $2.54(1.68-3.83)$ & $<0.001$ \\
\hline Fasting plasma glucose, per $10 \mathrm{mg} / \mathrm{dL}$ & $1.02(0.99-1.04)$ & 0.115 \\
\hline Lp(a) corrected LDL-C, per mg/dL & $1.01(1.00-1.01)$ & 0.009 \\
\hline $\mathrm{eGFR}, \mathrm{mL} / \mathrm{min} / 1.73 \mathrm{~m}^{2}$ & $0.98(0.97-0.99)$ & $<0.001$ \\
\hline Mean HbAıc (per $1 \%$ increment) & $1.24(1.12-1.36)$ & $<0.001$ \\
\hline Diabetic nephropathy (yes vs. no) & $1.49(1.09-2.03)$ & 0.013 \\
\hline \multicolumn{3}{|l|}{$\operatorname{Lp}(a)$} \\
\hline 1st Quartile & 1.00 & \\
\hline 2nd Quartile & $0.97(0.61-1.56)$ & 0.910 \\
\hline 3rd Quartile & $1.75(1.15-2.65)$ & 0.009 \\
\hline 4th Quartile & $2.37(1.59-3.55)$ & $<0.001$ \\
\hline
\end{tabular}

CI, confidence interval; ACEi, angiotensin converting enzyme inhibitor; ARB, angiotensin receptor blocker; Lp(a), lipoprotein(a); LDL-C, low density lipoprotein cholesterol; eGFR, estimated glomerular filtration rate; HbA1c, glycated hemoglobin.

Table 4. Multivariable Cox proportional hazards model for cardiovascular disease by levels of $\operatorname{Lp}(\mathrm{a})$

\begin{tabular}{|c|c|c|c|c|}
\hline \multirow{2}{*}{ Variable } & \multicolumn{4}{|c|}{ Hazard ratio (95\% CI) } \\
\hline & Model 1 & Model 2 & Model $_{3}$ & Model 4 \\
\hline \multicolumn{5}{|l|}{$\operatorname{Lp}(\mathrm{a})$} \\
\hline ıst Quartile & 1.00 & 1.00 & 1.00 & 1.00 \\
\hline 2nd Quartile & $0.81(0.51-1.31)$ & $0.77(0.48-1.25)$ & $0.77(0.48-1.25)$ & $0.81(0.50-1.32)$ \\
\hline 3rd Quartile & $1.50(0.98-2.29)$ & $1.40(0.91-2.15)$ & $1.38(0.90-2.13)$ & $1.46(0.95-2.25)$ \\
\hline 4th Quartile & $2.00(1.33-3.01)$ & $1.85(1.22-2.80)$ & $1.74(1.15-2.65)$ & $1.92(1.26-2.92)$ \\
\hline$p$ for trend & $<0.001$ & $<0.001$ & 0.001 & $<0.001$ \\
\hline
\end{tabular}

Multivariable Cox proportional hazards models were adjusted for the following covariates: model 1 (sex, age); model 2 (model 1 + diabetes duration, hypertension, smoking, body mass index, mean glycated hemoglobin, and diabetic nephropathy); model 3 (model 2 + estimated glomerular filtration rate, Lp(a) corrected low density lipoprotein cholesterol); model 4 (model $3+$ insulin, angiotensin converting enzyme inhibitor/angiotensin receptor blockers, statin, and acetylsalicylic acid).

Lp(a), lipoprotein(a); CI, confidence interval. 
death and CAD event) as well as the extent of CAD [22]. Kwon et al. [22] reported that Lp(a) was a significant predictor of major adverse cardiovascular events in patients with symptomatic CAD (HR, 1.773; 95\% CI, 1.194 to 2.634; $p=0.005)$. However, few prospective studies have been conducted to determine the association between $\mathrm{Lp}(\mathrm{a})$ and CVD in type 2 diabetes.

In addition, several studies have shown inconsistent findings in the association between $\mathrm{Lp}(\mathrm{a})$ concentration and the development of CVD in type 2 diabetes [12-17]. $\mathrm{Lp}(\mathrm{a})$ was inversely associated with the new onset of type 2 diabetes after adjusting for established CAD risk factors and $\mathrm{HbAlc}[11]$. A meta-analysis of two prospective studies showed that type 2 diabetes may attenuate the association of Lp(a) with CVD [12]. On the contrary, a long-term prospective study showed that an increased $\mathrm{Lp}(\mathrm{a})$ level was associated with an increased risk of CVD in women with diabetes [13]. A 10-year follow-up study reported a positive relationship between $\mathrm{Lp}$ (a) concentration and CVD in type 2 diabetes [14]. The different results of these studies might probably be due to different confounding variables in study design (prospective or cross-sectional, follow-up duration, and sample size), population characteristics (age, sex, drug use, and ethnicity), disease status (duration of diabetes, comorbidity), confounding variables (lipid abnormalities, thrombosis risk factors), or the Lp(a) assay method [12-17]. For example, Nurses' Health Study was conducted only in the women [13] or small number numbers of participants (n $=100$ ) and were not controlled for treatments that could influence cardiovascular risk [14]. A study by Hiraga et al. [16] was conducted for only 2.2 to 3.1 years, and $L p(a)$ was semi-quantified by an electrophoretic method that distinguished high from low serum $L p(a)$ at the level of $20 \mathrm{mg} / \mathrm{dL}$. In the study by Qi et al. [12], they did not exclude non-fasting samples, and the proportion of postmenopausal hormone users was $24.6 \%$. However, there were no studies in which mean HbAic was adjusted for fasting $\mathrm{Lp}(\mathrm{a})$ level as a confounding variable.

In addition, long-term glycemic control status is an important risk factor for the development of diabetic macrovascular complications. We followed our patients for more than 11 years and measured the HbAic level regularly. In an univariable analysis, the RR of a mean HbAlc (per 1\% increment) for a future CVD was 1.24 (95\% CI, 1.12 to 1.36 ; $p<0.001$ ). However, in a multivari- able analysis that was adjusted for a mean $\mathrm{HbAic}$ as a covariate, Lp(a) level (4th quartile) was still an independent risk factor for CVD development. Our hypothesis is that having $\operatorname{Lp}(\mathrm{a})$ levels in the highest quartile is an important biomarker for the prediction of CVD in patients with type 2 diabetes, independent of the patient's glycemic control status.

Previous meta-analyses and cohort studies consistently showed that significant increases in CVD risk were seen if $\mathrm{Lp}$ (a) levels exceeded the threshold of 30 to 50 $\mathrm{mg} / \mathrm{dL}$ or in the highest tertile or quintile in the general population [10,13,23-26]. In this long-term prospective cohort study, we suggested that an elevated Lp(a) level was an independent predictive risk factor for future development of CVD in type 2 diabetes. After adjusting for multiple confounding variables, we found a substantially higher HR of 1.92 (95\% CI, 1.26 to 2.92; $p<0.001$ ) for the top quartile compared with the bottom quartile. Similarly, Shai et al. [13] reported that diabetic women in the 5 th quintile of $\mathrm{Lp}(\mathrm{a})$ had a relative risk for $\mathrm{CAD}$ of 1.95 (95\% CI, 1.07 to 3.56; $p$ for trend $=0.035$ ) compared with those in the 1st quintile $\mathrm{Lp}(\mathrm{a})$.

There are significant differences in Lp(a) levels among different ethnic groups [27-31]. Recently, Guan at el. [25] reported a different cutoff of $\mathrm{Lp}(\mathrm{a})$ for CVD in other ethnic groups. The Lp(a) HR by the $50 \mathrm{mg} / \mathrm{dL}$ cut point was 1.69 (95\% CI, 1.03 to $2.76 ; p=0.037$ ) in blacks, 1.82 (95\% CI, 1.15 to $2.88 ; p=0.010$ ) in whites, and 2.37 in Hispanics (95\% CI, 1.17 to $4.78 ; p=0.017$ ). The lower Lp(a) cut point of $30 \mathrm{mg} / \mathrm{dL}$ was significant only in blacks (HR, 1.87; 95\% CI, 1.08 to 3.21; $p=0.024$ ). Chinese Americans showed no significant cut off for $\operatorname{Lp}(\mathrm{a})$ [25]. Prospective studies reported that there were no significant gender-related differences in $\mathrm{Lp}(\mathrm{a})$ concentrations [30-31]. However in other studies, $L \mathrm{p}(\mathrm{a})$ levels in females were higher than that in males $[8,29,32]$. A cohort study from Taiwan reported that geometric means of Lp(a) level were higher in females, and the difference was significant for the 45 to 54 years age group [32]. When considering the above results, we suggest that quartile or quintile range of Lp(a) levels might be more useful as a prognostic predictor for CVD compared with the previously reported cutoff value of 30 or $50 \mathrm{mg} / \mathrm{dL}$.

The pathogenic mechanism between $\mathrm{Lp}(\mathrm{a})$ and CVD remains unknown. $L p(a)$ is present in the arterial wall at the sites of atherosclerosis in humans and is implicated 
in the process of atherogenesis [33-35]. In addition, Lp(a) carries oxidized phospholipids associated with vascular inflammation and progression of atherosclerosis [35,36].

Despite its evident role as a risk factor for CVD, there are some limitations for the clinical application of $\mathrm{Lp}(\mathrm{a})$. The Lp(a) assay has an extremely wide range of plasma levels from 0.1 to $300 \mathrm{mg} / \mathrm{dL}$ with a skewed deviation, and there are differences in levels among different ethnic populations [27-31]. In addition, Lp(a) levels are not influenced by lifestyle modification [6], and there is no proper treatment for lowering Lp(a) level [37]. Therefore, Lp(a) measurement could not be strongly recommended as a routine clinical practice in the general population. However, Lp(a) screening should be considered in subjects with higher CVD risk such as patients with type 2 diabetes.

There are some limitations in this study. First, this study was performed at single center in one ethnic group. As described above, $L p(a)$ concentrations vary significantly among different ethnic groups [27-31]. Generally, independent of isoform variations, Chinese and Asian populations are known to have lower plasma Lp(a) levels than those of Caucasians [28]. Second, we could not measure the apo(a) isoform and LPA genotypes at baseline. However, according to the results of the Bruneck Study, apo(a) isoforms and LPA genotype did not improve the prognostic value of the $\mathrm{Lp}(\mathrm{a})$ concentration [38]. Therefore, a well-standardized quantitative Lp(a) assay without measuring the isoform size may be enough to predict the risk of CVD in routine clinical practice [39]. Third, because of a lack of consensus regarding the standard assay method for Lp(a) measurement, we analyzed Lp(a) level as a quartile range, rather than as cutoff values. The expression of $L p(a)$ level as a quartile range is not a standardized approach, therefore, it is difficult to generalize and is subject to certain biases.

In spite of some limitations, our study still has strengths, compared to the previous studies as followings. (1) All of the blood samples were collected from fasting patients and the serum Lp(a) level was measured on a daily basis without freezing. (2) This is a long term prospective cohort study. (3) The available clinical parameters related to CVD risk factors were adjusted as various confunding factors, including mean $\mathrm{HbArc}$ during the study follow-up.

In conclusion, we demonstrated that an elevated $\mathrm{Lp}(\mathrm{a})$ level was an independent predictive risk factor for CVD in type 2 diabetes. Lp(a) screening could be a useful biomarker for detecting individuals with a high CVD risk in type 2 diabetes. Quartile or quintile range of $L p(a)$ levels rather than cutoff values may be more useful as a prognostic risk factor for CVD. At the same time, other cardiovascular risk factors should be treated more intensively in type 2 diabetes patients with high $\mathrm{Lp}(\mathrm{a})$ levels. Additionally, the pathogenic mechanism of the causal relationship between Lp(a) and CVD requires further clarification.

\section{KEY MESSAGE}

1. Lipoprotein(a) (Lp[a]) level was an independent predictive risk factor for cardiovascular disease (CVD) in type 2 diabetes.

2. Quartile or quintile range of $\operatorname{Lp}(\mathrm{a})$ levels may be useful as a prognostic risk factor for CVD.

3. As there is no proper treatment for lowering $\mathrm{Lp}$ (a) level, other cardiovascular risk factors should be treated more intensively in type 2 diabetic patients with high Lp(a) levels.

\section{Conflict of interest}

No potential conflict of interest relevant to this article was reported.

\section{REFERENCES}

1. Uusitupa MI, Niskanen LK, Siitonen O, Voutilainen E, Pyorala K. Ten-year cardiovascular mortality in relation to risk factors and abnormalities in lipoprotein composition in type 2 (non-insulin-dependent) diabetic and non-diabetic subjects. Diabetologia 1993;36:1175-1184.

2. Almdal T, Scharling H, Jensen JS, Vestergaard H. The independent effect of type 2 diabetes mellitus on ischemic heart disease, stroke, and death: a population-based study of 13,000 men and women with 20 years of follow-up. Arch Intern Med 2004;164:1422-1426.

3. Rhee SY, Chon S, Kwon MK, et al. Prevalence of chronic complications in Korean patients with type 2 diabetes mellitus based on the Korean national diabetes program. Diabetes Metab J 2011;35:504-512.

4. Stevens RJ, Kothari V, Adler AI, Stratton IM; United King- 
dom Prospective Diabetes Study (UKPDS) Group. The UKPDS risk engine: a model for the risk of coronary heart disease in type II diabetes (UKPDS 56 ). Clin Sci (Lond) 2001;101:671-679.

5. National Cholesterol Education Program (NCEP) Expert Panel on Detection, Evaluation, and Treatment of High Blood Cholesterol in Adults (Adult Treatment Panel III). Third report of the National Cholesterol Education Program (NCEP) expert panel on detection, evaluation, and treatment of high blood cholesterol in adults (Adult Treatment Panel III) final report. Circulation 2002;106:3143-3421.

6. Utermann G. Lipoprotein (a). In: Scriver CR, Beaudet AL, Sly WS, Valle D, eds. The Metabolic and Molecular Bases of Inherited Disease. 2001 ed. New York: Mc-Graw-Hill, Medical Publishing Division, 2006:2753-2787.

7. Lamon-Fava S, Diffenderfer MR, Marcovina SM. Lipoprotein(a) metabolism. Curr Opin Lipidol 2014;25:189-193.

8. Emerging Risk Factors Collaboration, Erqou S, Kaptoge $\mathrm{S}$, et al. Lipoprotein(a) concentration and the risk of coronary heart disease, stroke, and nonvascular mortality. JAMA 2009;302:412-423.

9. Kamstrup PR, Tybjaerg-Hansen A, Steffensen R, Nordestgaard BG. Genetically elevated lipoprotein(a) and increased risk of myocardial infarction. JAMA 2009;301:23312339.

10. Danesh J, Collins R, Peto R. Lipoprotein(a) and coronary heart disease: meta-analysis of prospective studies. Circulation 2000;102:1082-1085.

11. Kamstrup PR, Nordestgaard BG. Lipoprotein(a) concentrations, isoform size, and risk of type 2 diabetes: a Mendelian randomisation study. Lancet Diabetes Endocrinol 2013;1:220-227.

12. Qi Q, Workalemahu T, Zhang C, Hu FB, Qi L. Genetic variants, plasma lipoprotein(a) levels, and risk of cardiovascular morbidity and mortality among two prospective cohorts of type 2 diabetes. Eur Heart J 2012;33:325-334.

13. Shai I, Schulze MB, Manson JE, Stampfer MJ, Rifai N, Hu FB. A prospective study of lipoprotein(a) and risk of coronary heart disease among women with type 2 diabetes. Diabetologia 2005;48:1469-1476.

14. Hernandez C, Francisco G, Chacon P, Simo R. Lipoprotein(a) as a risk factor for cardiovascular mortality in type 2 diabetic patients: a 10-year follow-up study. Diabetes Care 2005;28:931-933.

15. Murase T, Okubo M, Amemiya-Kudo M, Ebara T, Mori Y.
Impact of elevated serum lipoprotein (a) concentrations on the risk of coronary heart disease in patients with type 2 diabetes mellitus. Metabolism 2008;57:791-795.

16. Hiraga T, Kobayashi T, Okubo M, et al. Prospective study of lipoprotein(a) as a risk factor for atherosclerotic cardiovascular disease in patients with diabetes. Diabetes Care 1995;18:241-244.

17. Ginier P, Deedwania P. Lipoprotein(a) in patients who have non-insulin-dependent diabetes with and without coronary artery disease. Endocr Pract 1997;3:276-280.

18. Kang HM, Lee YJ, Kim DJ. The association of self-reported coronary heart disease with diabetes duration in Korea. Diabetes Metab J 2012;36:350-356.

19. Ko SH, Kwon HS, Kim DJ, et al. Higher prevalence and awareness, but lower control rate of hypertension in patients with diabetes than general population: the fifth Korean National Health and Nutrition Examination Survey in 2011. Diabetes Metab J 2014;38:51-57.

20. Levey AS, Stevens LA, Schmid CH, et al. A new equation to estimate glomerular filtration rate. Ann Intern Med 2009;150:604-612.

21. Kronenberg F, Lingenhel A, Lhotta K, et al. Lipoprotein(a)- and low-density lipoprotein-derived cholesterol in nephrotic syndrome: impact on lipid-lowering therapy? Kidney Int 2004;66:348-354.

22. Kwon SW, Lee BK, Hong BK, et al. Prognostic significance of elevated lipoprotein(a) in coronary artery revascularization patients. Int J Cardiol 2013;167:1990-1994.

23. Nordestgaard BG, Chapman MJ, Ray K, et al. Lipoprotein(a) as a cardiovascular risk factor: current status. Eur Heart J 2010;31:2844-2853.

24. Kostner GM, Avogaro P, Cazzolato G, Marth E, Bittolo-Bon G, Qunici GB. Lipoprotein Lp(a) and the risk for myocardial infarction. Atherosclerosis 1981;38:51-61.

25. Guan W, Cao J, Steffen BT, et al. Race is a key variable in assigning lipoprotein(a) cutoff values for coronary heart disease risk assessment: the multi-ethnic study of atherosclerosis. Arterioscler Thromb Vasc Biol 2015;35:996-1001.

26. Kamstrup PR, Tybjaerg-Hansen A, Nordestgaard BG. Extreme lipoprotein(a) levels and improved cardiovascular risk prediction. J Am Coll Cardiol 2013;61:1146-1156.

27. Guyton JR, Dahlen GH, Patsch W, Kautz JA, Gotto AM Jr. Relationship of plasma lipoprotein Lp(a) levels to race and to apolipoprotein B. Arteriosclerosis 1985;5:265-272.

28. Sandholzer C, Hallman DM, Saha N, et al. Effects of the apolipoprotein(a) size polymorphism on the lipopro- 
tein(a) concentration in 7 ethnic groups. Hum Genet 1991;86:607-614.

29. Brown SA, Hutchinson R, Morrisett J, et al. Plasma lipid, lipoprotein cholesterol, and apoprotein distributions in selected US communities: the Atherosclerosis Risk in Communities (ARIC) Study. Arterioscler Thromb 1993;13:1139-1158.

30. Jenner JL, Ordovas JM, Lamon-Fava S, et al. Effects of age, sex, and menopausal status on plasma lipoprotein(a) levels: the Framingham Offspring Study. Circulation 1993;87:1135-1141.

31. Marcovina SM, Albers JJ, Jacobs DR Jr, et al. Lipoprotein[a] concentrations and apolipoprotein[a] phenotypes in Caucasians and African Americans: the CARDIA study. Arterioscler Thromb 1993;13:1037-1045.

32. Chien KL, Lee YT, Sung FC, Su TC, Hsu HC, Lin RS. Lipoprotein (a) level in the population in Taiwan: relationship to sociodemographic and atherosclerotic risk factors. Atherosclerosis 1999;143:267-273.

33. Nielsen LB, Gronholdt ML, Schroeder TV, Stender S, Nordestgaard BG. In vivo transfer of lipoprotein(a) into human atherosclerotic carotid arterial intima. Arterioscler
Thromb Vasc Biol 1997;17:905-911.

34. Baldo G, Giunco S, Kontothanassis D, Baiocchi MR, Valerio A, Frego M. Different apoprotein(a) isoform proportions in serum and carotid plaque. Atherosclerosis 2007;193:177-185.

35. Kamstrup PR. Lipoprotein(a) and ischemic heart disease: a causal association? A review. Atherosclerosis 2010;211:1523.

36. Tsimikas S, Bergmark C, Beyer RW, et al. Temporal increases in plasma markers of oxidized low-density lipoprotein strongly reflect the presence of acute coronary syndromes. J Am Coll Cardiol 2003;41:360-370.

37. Kronenberg F. Epidemiology, pathophysiology and therapeutic implications of lipoprotein(a) in kidney disease. Expert Rev Cardiovasc Ther 2004;2:729-743.

38. Willeit P, Kiechl S, Kronenberg F, et al. Discrimination and net reclassification of cardiovascular risk with lipoprotein(a): prospective 15-year outcomes in the Bruneck Study. J Am Coll Cardiol 2014;64:851-86o.

39. Kostner KM, Marz W, Kostner GM. When should we measure lipoprotein (a)? Eur Heart J 2013;34:3268-3276. 
Supplementary Table 1. Multivariable Cox proportional hazards model for heart disease and stroke by levels of Lp(a)

\begin{tabular}{|c|c|c|c|c|}
\hline \multirow{2}{*}{ Variable } & \multicolumn{4}{|c|}{ Hazard ratio (95\% CI) } \\
\hline & Model 1 & Model 2 & Model $_{3}$ & Model $_{4}$ \\
\hline \multicolumn{5}{|c|}{ Stroke $(\mathrm{n}=97 / 833,11.6 \%)$} \\
\hline \multicolumn{5}{|c|}{$\mathrm{Lp}(\mathrm{a})$} \\
\hline 1st Quartile & 1.00 & 1.00 & 1.00 & 1.00 \\
\hline 2nd Quartile & $0.80(0.41-1.58)$ & $0.78(0.39-1.54)$ & $0.76(0.38-1.51)$ & $0.81(0.41-1.62)$ \\
\hline 3rd Quartile & $1.08(0.57-2.04)$ & $0.98(0.51-1.88)$ & $0.98(0.51-1.88)$ & $1.03(0.54-1.98)$ \\
\hline 4th Quartile & $2.11(1.19-3.75)$ & $1.01(1.22-3.27)$ & $1.86(1.03-3.36)$ & $2.10(1.16-3.80)$ \\
\hline$p$ for trend & & & & $<0.001$ \\
\hline \multicolumn{5}{|c|}{ Heart disease $(n=114 / 833,13.7 \%)$} \\
\hline \multicolumn{5}{|l|}{$\operatorname{Lp}(\mathrm{a})$} \\
\hline 1st Quartile & 1.00 & 1.00 & 1.00 & 1.00 \\
\hline 2nd Quartile & $0.77(0.40-1.48)$ & $0.74(0.38-1.43)$ & $0.73(0.38-1.42)$ & $0.78(0.40-1.53)$ \\
\hline 3rd Quartile & $1.96(1.13-3.41)$ & $1.85(1.06-3.25)$ & $1.84(1.04-3.23)$ & $1.96(1.11-3.46)$ \\
\hline 4th Quartile & $2.01(1.15-3.52)$ & $1.70(0.96-3.00)$ & $1.72(0.97-3.06)$ & $1.85(1.04-3.30)$ \\
\hline$p$ for trend & & & & $<0.001$ \\
\hline
\end{tabular}

Lp(a), lipoprotein(a); CI, confidence interval. 


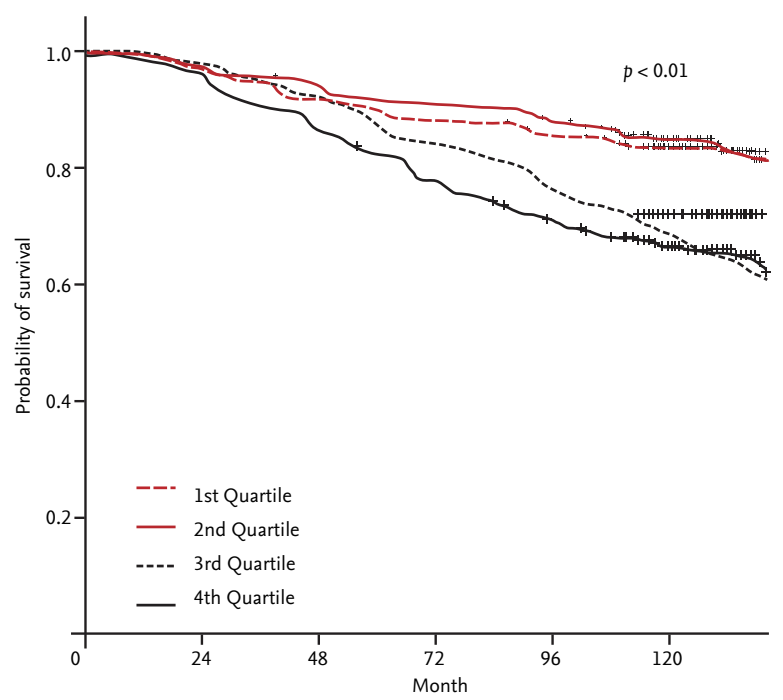

Supplementary Figure 2. Kaplan-Meier curve of cardiovascular disease events according to the quartiles of lipoprotein(a). 